\title{
Environmental Temperature Problem Identified
}

National Cancer Institute

\section{Source}

National Cancer Institute. Environmental Temperature Problem Identified. NCI

Thesaurus. Code C92134.

Device performance was affected by the temperature, or changes in temperature, of the environment in which it was used. 